\title{
Fibonacci, Yablo and the Cassationist Approach to Paradox
}

\author{
LAURENCE GOLDSTEIN
}

\begin{abstract}
A syntactically correct number-specification may fail to specify any number due to underspecification. For similar reasons, although each sentence in the Yablo sequence is syntactically perfect, none yields a statement with any truth-value. As is true of all members of the Liar family, the sentences in the Yablo sequence are so constructed that the specification of their truth-conditions is vacuous; the Yablo sentences fail to yield statements. The 'revenge' problem is easily defused. The solution to the semantical paradoxes offered here revives the mediaeval cassatio approach, one that largely disappeared due to its incomprehending rejection by influential contemporary writers such as William Shyreswood and Thomas Bradwardine. The diagnosis readily extends to the set-theoretic paradoxes.
\end{abstract}

\section{Variations on Fibonacci}

The Fibonacci series $1,1,2,3,5,8,13,21,34,55 \ldots$ has many beautiful and fascinating properties. It is an infinite series of numbers in which each term is the sum of its two immediate predecessors: Fib[i] $=$ Fib[i-2] + Fib[i-1]. Yablo's Paradox, which is a hard, or 'strengthened' version of the Liar, consists of an infinite sequence of sentences, each saying of all its successors that they are not true (Yablo 1985; 1993). I hope to show that there is an interesting connection between the series and the sequence. There is nothing paradoxical about the series, but the sequence is paradoxical, and I want to claim that, once the connection is made, the paradox is swiftly solved. The solution, of course, carries over to simpler and standard versions of the Liar.

Each member of the Fibonacci series 'remembers', and sums, its immediate two predecessors. Series constructed on the same general principle, but with better memories, are 
invariably less interesting than Fibonacci's original. In the 'total recall' series, where each member sums all of its predecessors, the first two members are identical and any member after that is simply twice its predecessor. One can also have 'soothsayer' or 'forward-looking' variants of Fibonacci. For example, the series in which each member is the sum of its immediate two successors is just the Fibonacci series in reverse.

One way to manufacture an extremely boring variant of Fibonacci is to go Boolean, using a binary arithmetic in which addition ('+') is defined by the following matrix: 


$\begin{array}{ccc}\mathrm{x} & \mathrm{y} & \mathrm{x}+\mathrm{y} \\ 1 & 1 & 0 \\ 1 & 0 & 1 \\ 0 & 1 & 1 \\ 0 & 0 & 0\end{array}$

The Boolean Fibonacci, in which each member is the Boolean sum (as defined above) of its immediate two predecessors, is:

1

0

1

1

0

Unlike Fibonacci's series, or the related 'Golden String', ${ }^{1}$ this series is repetitive — it is an infinite repetition of a three-digit pattern. 


\section{From arithmetic to logic}

Let us represent the classical truth-values 'true' and 'false' by the binary digits ' 0 ' and ' 1 ' respectively. Then val $(\sim \mathrm{p})=1-\mathrm{val}(\mathrm{p})$, and classical disjunction translates as Boolean multiplication, val $(p \vee q)=\operatorname{val}(p) x$ val $(q)$. All of the classical truth-functional connectives can be interpreted as Boolean functions. Since classical semantics has this arithmetic interpretation, we might entertain the thought that there is some counterpart in classical logic to the Boolean Fibonacci. Consider, then, an infinite sequence of statements, each one of which reads 'Both of the next two statements are not true'. We shall call this the Fibber2acci sequence (the ' 2 ' is there as a reminder that each member says something about the next $t w o$ statements). Let ' $\mathrm{S}_{\mathrm{i}}$ ' abbreviate the name of the ith statement in the sequence. Now construct a value table for the sequence (I show only the first five rows):

\begin{tabular}{|r|r|r|r|r|}
\hline $\mathrm{S}_{1}$ & 0 & & & \\
\hline $\mathrm{S}_{2}$ & 1 & 0 & 1 & 0 \\
\hline $\mathrm{S}_{3}$ & 1 & 1 & 0 & 0 \\
\hline $\mathrm{S}_{4}$ & 0 & 1 & 1 & $*$ \\
\hline $\mathrm{S}_{5}$ & 1 & 0 & 1 & \\
\hline
\end{tabular}

The first point to note is that, where any statement $S_{i}$ has the value 0 (i.e., 'true') this requires that both $S_{i+1}$ and $S_{i+2}$ are not true; both have value 1. Further, since, under this assumption, $\mathrm{S}_{\mathrm{i}+1}$ is not true, and what it says is that the two statements succeeding it are not true, it follows that at least one of those statements must be true, and since, as we have already worked out, $S_{i+2}$ is not true, it must be $S_{i+3}$ that is true. And now this reasoning repeats: since $S_{i+3}$ is true, the two statements following it are untrue etc.. Thus, it is easy to trace the consequence of any statement in the sequence being true. If some statement in the sequence (say $S_{1}$ ) is not 
true, i.e., that it is not the case that the two statements following it are not true, then we have to consider the three possibilities indicated by the three branches at the right of the table. However, the rightmost branch is not a live option since, as we have already shown, a statement with value 0 must be followed by two with value 1 . Therefore there are just three columns in the table, and each displays the '011' pattern. So the Fibber2acci has a Boolean Fibonacci evaluation table. This result, though pretty, has no deep theoretical significance, but it is worth remarking here on an oddity to which we shall return later. Although, all the token sentences in the Fibber2acci are identical and, in a clear sense, each appears to say the same thing (viz. just that both of the statements following it are not true), the evaluation table indicates that it is not the case that all of the statements in the series have the same truthvalue.

\section{The Yablo sequence}

For a more far-reaching conclusion, we now turn to Yablo's sequence of sentences. It is a total foresight sequence, since each of the constituent sentences is about all of the subsequent ones:

$\mathrm{Y}_{1}$ : For all integers $\mathrm{n}>1, \mathrm{Y}_{\mathrm{n}}$ is not true.

$\mathrm{Y}_{2}$ : For all integers $n>2, Y_{n}$ is not true.

$Y_{3}$ : For all integers $n>3, Y_{n}$ is not true. 
A typical member $Y_{k}$ is 'For all integers $n>k, Y_{n}$ is not true'. The Yablo sequence is paradoxical. This may be proved formally and can be illustrated graphically by means of a value table (only the first ten rows of which are here displayed):

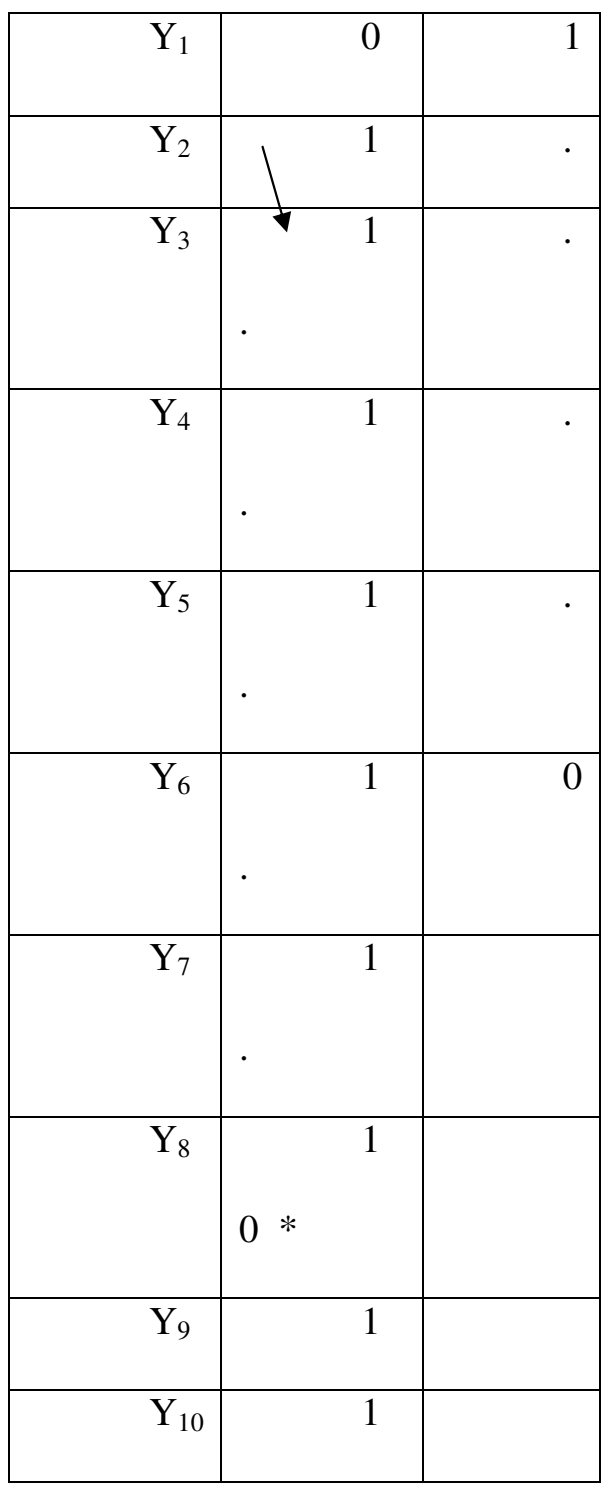

If $Y_{1}$ has value 0 ('true'), as shown in the middle column of the first row, then all subsequent statements in the sequence must be not true. In particular, $Y_{2}$ is not true. But since $Y_{2}$ says that all statements subsequent to it are not true, at least one of them (e.g. $\left.\mathrm{Y}_{8}\right)$ must be true and that is inconsistent with the result already obtained that, under the assumption that the first 
statement in the sequence is true, all of the rest must be not true. Hence, by reductio, $\mathrm{Y}_{1}$ is not true. However, if $Y_{1}$ is false, as shown in the last column of the first row, that means that at least one statement in the sequence (e.g. $\mathrm{Y}_{6}$ ) must be true. But now we can reason about that statement's truth and derive a contradiction in just the same way as we reasoned to a contradiction from the assumption that $Y_{1}$ was true. Hence, the assumption that $Y_{1}$ is false equally cannot be sustained.

We talk about Yablo's Paradox, but it should be pointed out that all we have so far shown is that $Y_{1}$ cannot be true and also that $Y_{1}$ cannot be false. Now there would be a paradox only if we had independent grounds for saying that $Y_{1}$ must be true or false, as classical semantics requires. There certainly seem to be such grounds, because all sentences in the Yablo sequence are well-formed, and each is intelligible - after all, each appears just to say that all subsequent statements in the sequence are not true. It is because we understand the sentences in the sequence that we can reason about them, infer contradiction and so on. However, that a sentence is meaningful does not entail that it can be used to make a truthvalued statement, ${ }^{2}$ and this may be the case with sentences in the Yablo sequence. Let us go back to the Fibonacci series to see if it can throw some light on our problem.

Earlier on, we pretended to give a proper characterization of the Fibonacci series. But that characterization was incomplete; all we said was that Fib[i] $=$ Fib[i-2] + Fib[i-1]. Clearly we cannot assign any numerical values to any member of the series unless we first specify Fib[1] and Fib[2], or at least first stipulate a value for at least two members of the series. For different stipulations, one obviously gets different series; without such stipulation all we have is a class of functions $\{\mathrm{f}: \mathrm{N} \rightarrow \mathrm{N}$ If(n) $=\mathrm{f}(\mathrm{n}-2)+\mathrm{f}(\mathrm{n}-1)\}$, no particular series but only the characterization of a generic series. Without the specification of values for at least two members, there is no grounding; no particular series is specified. Now, the Yablo sequence is a counterpart of the total foresight variant of the reverse Fibonacci - any member of the 
sequence purports to say something about all the subsequent members. The truth-value of any member of this sequence, if grounded, is grounded on the truth-values of subsequent members, since it asserts the untruth of each of them. But the truth-value of each of these is, in turn, inherited from the truth-values of members subsequent to it. Since there is nothing that fixes the truth-value of any member of the sequence, the reasonable conclusion to draw is that no member of the Yablo sequence has a truth-value ${ }^{3}$ (or that each has the value 'undefined' or 'GAP' ${ }^{4}$ ), just as no member of our underspecified (generic) Fibonacci has a numerical value.

One could write out the standard Fibonacci series in the following way:

1

1

The sum of the two preceding numbers

The sum of the two preceding numbers

The sum of the two preceding numbers

Each token noun-phrase in this series designates a definite number (and each designates a number different from that designated by the others). But, in the generic Fibonacci

The sum of the two preceding numbers

The sum of the two preceding numbers 
The sum of the two preceding numbers

each of the noun-phrases fails to designate any number. And, if we write out the Yablo sequence as follows:

Each succeeding statement is untrue.

Each succeeding statement is untrue.

Each succeeding statement is untrue.

we can likewise say that each of those token sentences fails to make a statement - fails to have a truth-value. Note that the failure to have a value (or to have the value 'GAP') is not a consequence of the fact that one can derive a contradiction from the Yablo sequence. No member of the infinite sequence

The next statement is true.

The next statement is true.

The next statement is true. 
has a truth-value, even though one could consistently ascribe the value 'true' (or the value 'false') to all of them. We have here a 'Buridan's Ass' type of situation - since, for any member of this sequence, there is no sufficient reason to ascribe it the value 'true' rather than 'false' and vice-versa, the proper course is to withhold the ascription of any truth-value. We do this not because of ignorance as to which truth-value to ascribe, but because it would be incorrect to ascribe any value. Given the correspondingly simple series

The same number as the next

The same number as the next

The same number as the next

nobody would say that there is a correct but unknown numerical value that each member of this series designates.

Perhaps the best way of seeing the Fibonacci-Yablo analogy is to compare, side by side, the reverse Yablo with the total recall Fibonacci

All the preceding statements are untrue. The sum of all preceding numbers

All the preceding statements are untrue. The sum of all preceding numbers

All the preceding statements are untrue. The sum of all preceding numbers 
No statements and no numbers are defined, due to underspecification. It may be thought strange that something that looks like a statement or looks like a form of words designating a number should, nevertheless, not be one. ${ }^{5}$ But look at the phrase 'The natural number that is the successor of itself'. There is clearly no number that it designates, despite the fact that it 'calls' itself a number. The natural numbers can be defined by the Peano axioms, and it is an immediate consequence of those axioms that no number can be its own successor. Equally clearly, though containing the words 'the statement', there is no statement designated by the phrase 'The statement that is true if an only if it is false'. This is because it is definitional of 'statement' that no statement is both true and false. ${ }^{6}$

A way of seeing that the Strengthened Liar sentence fails to make any statement is as follows: Take ' $\mathrm{J}$ ' to be the name of some statement. Then the statement ' $\mathrm{J}$ is not true' obviously has a truth-value different from (classically: opposite to) that of J. Therefore by (contraposition of) the law of the identity of indiscernibles, we cannot identify $\mathrm{J}$ with ' $\mathrm{J}$ is not true'; no such stipulation is admissible. The letter ' $\mathrm{J}$ ' was, of course, arbitrarily chosen. The conclusion we just drew can be formulated without the use of any particular letter - it is the conclusion that no statement can state of itself that it is not true. So, initial appearances to the contrary, 'This statement is not true' (where the indexical, in this version, assumes the reference-fixing function of the label) is not a statement; it states nothing; in particular, it does not state that it is not true.

There are therefore restrictions on the labeling of statements. ${ }^{7}$ The Liar is the simplest member of a large family of paradoxes, and it is easy to see, on a case-by-case basis, how the exposure of illegitimate labeling (or, as we shall see below, of other devices that achieve the same effect) dissolves these paradoxes. Consider the Card Paradox in which on one side of a card is written 'What is on the other side of this card is false' while what is 
written on the other side is 'What is on the other side of this card is true'. This paradox is often discussed in its 'label' variant:
(1) $\mathrm{S}_{1}: \mathrm{S}_{2}$ is false.
(2) $\mathrm{S}_{2}: \mathrm{S}_{1}$ is true.

(The A/B paradox discussed below is the truth-teller version of this.) We can legitimately assign the name ' $S_{1}$ ' to the statement ' $S_{2}$ is false' but, in so doing, we are stipulating that $S_{1}$ and $\mathrm{S}_{2}$ have opposite truth-values. Therefore, we are not free, in the same context, to assign the name ' $\mathrm{S}_{2}$ ' to ' $\mathrm{S}_{1}$ is true', for that stipulation would guarantee that $\mathrm{S}_{1}$ and $\mathrm{S}_{2}$ have the same truth-value. There is thus a restriction on what we can stipulate concerning the names ' $\mathrm{S}_{1}$ ' and ' $\mathrm{S}_{2}$ ' once the initial stipulation that ' $\mathrm{S}_{1}$ ' is to be the name of ' $\mathrm{S}_{2}$ is false' has been made. Another way of putting this would be to say that, after the initial assignment has been made, the names ' $\mathrm{S}_{1}$ ' and ' $\mathrm{S}_{2}$ ' are no longer free for indiscriminate use, if logical perspicuity is to be respected. This is not an ad hoc restriction, but a familiar one, the rationale for which is well understood. For example, in a natural deduction, we may make an inference from an existentially quantified proposition ' $(\exists x)(\ldots x \ldots)$ ' by instantiating the variable with a name. But then that name is no longer free for use as a substituend elsewhere in the deduction. A Social Security number, once assigned to someone, is not free for assigning to anybody else.

To see why it is reasonable to claim that both $S_{1}$ and $S_{2}$ lack content and fail to have any truth-value, one need only reflect that, since no independent information is given about their content, it has to be ascertained from the pair of sentences alone. Questions may be raised, and answered, about the physical makeup of $S_{1}$ and $S_{2} q u a$ inscriptions, or of the language in which $S_{1}$ and $S_{2}$ are framed. But it becomes clear that the question of the content 
of $S_{1}$ and $S_{2}$ is unanswerable. To specify their content one needs to set out the conditions under which they are true, and, as we shall see in section 5, this cannot be done.

To label a statement is to give a name to something that has a certain truth-value. Where there is a set of statements, each statement about some of the other statements in the set, the fact that a given statement has a certain truth-value will have consequences, sometimes tricky to track, for the truth-values of the other statements. If consistency is to be preserved, it may not be legitimate, as we have seen, to stipulate a certain label for a given statement. The Yablo sequence, as we have retailed it here, consists of a list of labeled sentences. Such a system of labeling produces an illegitimate stipulation; the case is no different in principle from the Liar and the Card paradoxes just considered, but the illegitimacy of the labeling is better disguised.

It is perfectly clear, however, that Yablo's paradox can be recast in a label-free form. There is the indexical variant - an infinite queue of people each of whom says 'What every person behind me is saying is untrue'. Quantifiers too are indexical; the extension of the phrase 'all the people in Room 101' varies from one occasion of use to another. In a quantificational variant of the simple Liar, the sentence 'All the statements on this board are false' is the only sentence appearing on the board in question. A quantificational variant of the Card Paradox consists of a pair of tokens written on an otherwise empty board thus:

All statements on this board are false.

All statements on this board are true.

Therefore an adequate cure for the Liar's ills must be more general than a diagnosis of 'illegitimate labeling'. 
We can consistently claim that, in this scenario, neither of the sentences yields a statement, while recognizing that if (say) the sentence ' $2+2=4$ ' were written below them on the board, then, in this new context, each of the sentences would yield a statement, the top two false. It is worth pausing here to say something about the bearers of truth and falsity. Up to this point, we have been calling them 'statements' and the preceding example illustrates why it is not tokens qua physical entities that are the bearers of truth. Before the addition of the sentence ' $2+2=4$ ', the top token on the board failed to yield a statement. With the new sentence added, that same token yields a false statement. Likewise, if I remove the tombstone bearing the inscription 'Here lies Thomas à Kempis' from the grave of Thomas à Kempis and erect it elsewhere, then a falsity appears and a truth disappears, even though the token inscription remains set in the same stone. This accounts for a difference with Gaifman (see note 5) on the question of what the bearers of truth and falsity are, and with Buridan (Hughes 1982) for whom, equally, truth-bearers are concrete tokens. Yet (as is widely recognized) it is not sentence-types that have truth-value. Consider the sequence:

The next statement is false.

The next statement is false.

Pigs can fly.

Clearly the first two statements, though constructed from the same type-sentence, differ in truth-value, so the bearers of truth cannot be type sentences. A statement is made by the use in a context of a token sentence (although a token of some grammatically acceptable sentence may fail, in a given context, to yield a statement); a statement is what is said by the use of a token on an occasion, and it is statements so construed (some philosophers call them 'propositions') that are the bearers of truth and falsity. 


\section{Chains and cycles}

A recurrent infinite chain paradox, i.e. a chain paradox containing infinitely repeated chunks, can be transformed into a finite paradox by snipping a representative chunk of the chain and forming a loop by bringing the two ends together (Goldstein 1999). Performing this operation on the Fibber2acci produces the Cyclical Fibber2acci. Consider the following three sentences

$\mathrm{H}: \mathrm{J}$ is not true and $\mathrm{K}$ is not true.

$\mathrm{J}: \mathrm{K}$ is not true and $\mathrm{H}$ is not true.

$\mathrm{K}: \mathrm{H}$ is not true and $\mathrm{J}$ is not true.

Inspection reveals that, if all of these sentences yield statements, one can consistently assign truth-values to each of them. Yet, if they possess truth-value, then they possess different truth-values: if any one is true, the other two must be false. But each of them says exactly the same thing about the other two. Therefore, by considerations of symmetry, if they possess truth-value, then they must each possess the same truth-value. ${ }^{8}$ It follows, then, that no classical truth-value can be assigned to $\mathrm{H}, \mathrm{J}$ or $\mathrm{K}$. Generalizing to the case of the finite nmembered sequence, we have the Cyclical Fibbernacci

$\mathrm{G}_{1}$ : For all integers $\mathrm{i}(1 \leq \mathrm{i} \leq \mathrm{n})$ except $\mathrm{i}=1, \mathrm{G}_{\mathrm{i}}$ is not true.

$\mathrm{G}_{2}$ : For all integers $\mathrm{i}(1 \leq \mathrm{i} \leq \mathrm{n})$ except $\mathrm{i}=2, \mathrm{G}_{\mathrm{i}}$ is not true.

$G_{n}$ : For all integers $i(1 \leq i \leq n)$ except $i=n, G_{i}$ is not true. 
If any member of this sequence has the value 'true', then all other members have the value 'not true'. But considerations of symmetry demand that each member have the same truthvalue. Hence, no member of the sequence has a classical truth-value (aliter: each has the value 'GAP'). As mentioned in section 3, refusal to assign a classical truth-value in cases like this is not a symptom of epistemic inadequacy. What we have here is, first, a proof that if the $\mathrm{G}_{\mathrm{i}}$ have truth-value (I take it that it would be absurd to suggest that some do, some don't), then the value of one differs from that of all the rest; second, a proof that if the $G_{i}$ have truthvalue, then the value of one is the same as that of all the rest. From which it follows that the $\mathrm{G}_{\mathrm{i}}$ do not have truth-value. They have meaning, of course, for, as previously noted, it is in virtue of understanding what such sentences mean that we can establish that tokens of them, as used in certain contexts, do not have truth-value. In David Kaplan's terms (1977), the (type-) sentences have character, but their tokens fail, in certain circumstances, to have content. Their lack of content may not be immediately apparent; similarly, it may take a few moments' thought to realize that the meaningful expression 'the successor of the integer denoted by this phrase' necessarily fails to denote.

In the light of the distinction between sentence and statement, on which we have been relying, the usual 'true / false / neither' trichotomy is best replaced, for the sake of perspicuity, by 'yields a true statement / yields a false statement / fails to yield a statement', predicable of token sentences relative to the contexts in which they are used. We have said that token sentences are the vehicles for statements; that we typically use token sentences to make statements, and that it is the statements so made that have content and truth-value. But there are occasions when, though we go through the motions of making a statement, no statement results (just as we can go through the motions of making a 'phone call but, when the number dialed is our own, no 'phone call results). At the beginning of this paper, we maintained that a series of numbers is properly defined by 'Fib[i] $=$ Fib[i-2] + Fib[i-1]', but 
subsequently revealed that that was just a pretence. On several occasions, we treated members of the Yablo sequence as if they were statements that 'say' something. But that this too is only make-believe can be further illustrated by a parable that illustrates why certain sentences must, under certain circumstances, fail to yield statements.

Consider a community of individuals — we shall call them the Cretins — each member of which utters just one sentence: 'All Cretins are total liars'. Nobody who utters such a sentence could, of course, be speaking truly, for that would entail that that very utterance was false. Yet it could be false, if some statement made by some Cretin were true. But all the Cretins say the same thing and, as we have just seen, what any of them says cannot be true. Hence no Cretin utterance can be true or false, a result in clear contradiction to the Principle of Bivalence. We seem to have here a reductio of the postulation of the Cretin community. Yet it is absurd to suggest that we can show, by pure logic alone, that a community of Cretins could not exist, so we have a paradox. There is nothing logically impossible about there being a bunch of people each of whom utters 'All Cretins are total liars', and nothing else. Logic cannot rule out the possibility of a Cretin community each member of which utters no more nor less than a token of that sentence. (You can test this by getting a few of your friends together and have them all recite, in unison, 'All of us are now uttering a falsehood'.)

But although logic cannot bargain out of existence the empirical possibility of Cretins uttering sentences, what it does rule out as logically impossible, is that the sentences uttered yield statements with truth-value, for it is precisely this assumption that is responsible for the contradiction noted above. ${ }^{9}$ The Cretin sentences are grammatically impeccable, yet, in the circumstances specified, they fail to yield statements. This is not a point specifically about spoken language. That the Cretins use their voices is incidental and inessential to our story and we could equally well have concocted a story about the tribe of Dumb Cretins, each of 
whom has the single belief — the single 'belief' - that what every other Dumb Cretin believes is false. No Dumb Cretin can even believe that all the beliefs held by Dumb Cretins are false. This may seem surprising until we recall the claim, now widely accepted in philosophy of mind circles, that the identity of a belief may be dependent, inter alia on the environment external to the believer. Consider person A holding the cretinous 'belief' that what he believes is false and that what B believes is false, where the only relevant feature of the environment external to $\mathrm{A}$ is person $\mathrm{B}$ holding the cretinous 'belief' that what she believes is false and that what A believes is false. A's cretinous 'belief' mentions B's, and depends for its content on her belief; but B's refers us back to A's, so no content can ever be found. Neither of these cretinous beliefs has an identifiable content.

Obviously, the Cretin paradox is a 'community' variant of the ancient paradox of Epimenides, the Cretan who tells St. Paul that all Cretans are liars (a paradox that itself morphs into the standard Eubulidean Liar if we suppose that a plague struck the Cretans, leaving Epimenides as the sole survivor) but, interestingly, it is also intimately related to Yablo's paradox. In fact, the Community Cretin paradox is just the non-exclusionary variant of the Cyclical Fibbernacci (the G-cycle set out above), and the latter, as we saw, is the general cyclical version of Yablo's paradox. Compare a sentence in the Q-cycle (below), which is a formal version of the Community Cretin, with a sentence in the G-cycle.

$\mathrm{Q}_{1}$ : For all integers $\mathrm{i}(1 \leq \mathrm{i} \leq \mathrm{n}), \mathrm{Q}_{\mathrm{i}}$ is not true.

$\mathrm{Q}_{2}$ : For all integers $\mathrm{i}(1 \leq \mathrm{i} \leq \mathrm{n}), \mathrm{Q}_{\mathrm{i}}$ is not true.

$\mathrm{Q}_{\mathrm{n}}$ : For all integers $\mathrm{i}(1 \leq \mathrm{i} \leq \mathrm{n}), \mathrm{Q}_{\mathrm{i}}$ is not true. 
It is easy to see that no truth-value can be consistently assigned to any of the $\mathrm{Q}_{\mathrm{i}}$. The tightest Q-cycle $(n=1)$ is simply the Strengthened Liar paradox. The tightest G-cycle, the Fibber1acci, is the strengthened version of Buridan's Sophism 8 (Hughes 1982). All of these paradoxes belong together in a family and, if the cassationist solution is good for one, it ought to be good for all. ('Cassatio' is the name of a proposed solution, popular in the early mediaeval period that was dismissed by influential authors such as William Shyreswood and Thomas Bradwardine (whose writings give no indication that they had given the view the serious attention that it merits). As P.V. Spade (1987) has documented, the proposed solution had virtually died out by the first quarter of the thirteenth century. According to the cassantes, attempted uses of paradoxical sentences to make statements are nullified, so that, although such sentences have meaning, no proposition or content gets to be expressed by them, and hence they lack truth-value.)

\section{The avoidance of revenge}

If all sentences in the Yablo sequence fail to yield statements and hence fail to have a truthvalue (or have the value 'GAP'), then all of them are not true. But the first member of the sequence, $\mathrm{Y}_{1}$, says that all the members subsequent to it are not true, so surely is $\mathrm{Y}_{1}$ is, after all, true! Yet the assumption that $\mathrm{Y}_{1}$ is true leads to contradiction. This is the kind of 'revenge' problem that always confronts solutions to Liar-type paradoxes that invoke truthvalue-gaps. The quick answer is that if, as we have argued, no sentence in the Yablo sequence makes a statement, then, in particular, $\mathrm{Y}_{1}$ does not state anything (correctly or incorrectly) and hence does not get assigned any truth-value. This answer is too quick to convince the uncommitted, and more needs to be said.

The Yablo sequence consists of an infinite number of labeled sentences, each one of which is perfectly grammatical and seems to be in perfect semantical order, yielding a 
statement referring unproblematically to all the sentences that follow it. We know what each sentence means; it is in virtue of knowing this that we can reason to a contradiction from the supposition that any of them yield true (or false) statements. So how can we claim that a person standing at the front of an infinite queue and saying 'They are all telling lies behind my back' or 'What each person behind me is saying is untrue' may not succeed in making a statement? Under what circumstances does a meaningful sentence uttered by a competent speaker not yield a statement?

It is important first to note that the distinction between a meaningful uttered sentence, and the proposition that a token sentence is used to express (or the statement that it makes) can be explained quite independently of any considerations about paradox. Think of a footpath, made of pebbles that each day get squished and squirted and scattered by the feet of innumerable walkers. Suppose that, at the end of one day, some pebbles, quite by chance, have ended up looking like a string of letters forming the sentence 'My brother is a doctor'. It would obviously be lunacy to ask 'Which brother are those pebbles talking about?' or 'By 'doctor' do they mean a physician or someone who has earned a Ph.D.?' By contrast, when I say to an injured companion 'My brother is a doctor', I am talking about my brother Hugh; by 'doctor' I mean, on this occasion, 'physician', by the sound 'is' I mean the present tense of the verb 'to be', not the 'is' of identity, and by 'a' the indefinite article. ${ }^{10}$ The words 'my' 'brother' 'is' 'a' 'doctor' in that order, no more constitute a unified proposition than do those words arranged in any other order. There is a categorical difference between sentence and statement or proposition.

Now consider the sentence 'The next statement is true'. As we saw in Section 3, considered simply as a set of marks, that token no more says anything than do the pebbles. If uttered, with appropriate intentions, by someone, then the resulting locution has some semantical properties, including speaker-meaning, but obviously it acquires the semantical 
property of having a truth-value only when the relevant next statement is made, for it inherits its truth-value from that latter statement. It would be ridiculous, before that time, to declare that a true statement had been made by the use of that sentence. Or a false one. There are no grounds for so declaring. Similarly, there is no temptation to suppose that any numerical values can be assigned in the generic forward-looking Fibonacci:

The sum of the numbers denoted by the following two noun-phrases

The sum of the numbers denoted by the following two noun-phrases

The sum of the numbers denoted by the following two noun-phrases

for, looking into the infinite beyond, there are no numbers designated, hence no two consecutive ones that can be summed.

Yet, while all reasonable people are quite happy to say that each member of the generic forward-looking Fibonacci series is a description that fails to describe any number, many may yet be reluctant to say that, in the corresponding Fibber2acci series (each member of which is 'Both of the next two statements are not true'), each member fails to make a statement. The difference seems to be that we are familiar with the phenomenon of expressions that fail to designate numbers (e.g. 'the real roots of' $x^{2}+x+1=0$ '), but, in the case of the Fibber2acci, each member seems to be saying something about the next two members and they are right there, just below it on the page, so it seems obvious that each member succeeds in making a statement. This appears to be even clearer if we formulate the Fibber2acci in such a way that reference is made only to sentences, viz., when each member is 'The following two sentences yield statements both of which are untrue'. In fact, Yablo's 
sequence can be expressed in a way tailor-made to resist the cassationist solution advocated here:

$\mathrm{YS}_{1}$ : For all integers $\mathrm{n}>1, \mathrm{YS}_{\mathrm{n}}$ either yields a falsehood or fails to make a statement. $\mathrm{YS}_{2}$ : For all integers $\mathrm{n}>2, \mathrm{YS}_{\mathrm{n}}$ either yields a falsehood or fails to make a statement. $\mathrm{YS}_{3}$ : For all integers $\mathrm{n}>3, \mathrm{YS}_{\mathrm{n}}$ either yields a falsehood or fails to make a statement.

We can consistently assign 'yields a truth' to $\mathrm{YS}_{1}$, and 'fails to make a statement' (aliter: 'does not possess a truth-value') to all the subsequent $\mathrm{YS}_{\mathrm{i}}$. This blocks the reasoning to a contradiction, for if $\mathrm{YS}_{2}$ fails to make a statement, then, of course, it does not make any truthvalue ascriptions to any of the members of the sequence subsequent to it; in particular it can make none that would be inconsistent with the values assigned to those members on the assumption that $\mathrm{YS}_{1}$ yields a truth.

Yet this is not a satisfying way with this strengthened version of Yablo's paradox. The first point to note is that this ascription of values almost entirely cedes the game to the cassationist, since all but one of the sentences in the Yablo sequence is acknowledged to yield no statement. But, further, considerations of symmetry militate against even this ascription of values to members of this sequence. For one could equally and consistently posit that $\mathrm{YS}_{217}$ yields a truth while all other members of the sequence lack truth-value. But clearly it is quite implausible to suggest that $\mathrm{YS}_{217}$ yields a truth while $\mathrm{YS}_{218}$ lacks a truth-value. No, for the Yablo sequence, the only reasonable assignment is 'lacks a truth-value' to all of its members. The YS version of the Yablo paradox fails to wreak revenge on the cassationist solution. 
Although the revenge problem can be defeated by denying truth-value to any of the $\mathrm{YS}_{\mathrm{i}}$, the victory is Pyrrhic unless we can explain why a sentence that looks, for all the world, as if it is being used to make a statement nevertheless fails to yield one. There is a real tension here. As we saw with the Fibber2acci sequence, all of its members have meaning and classical truth-values can be assigned to all of them, every 'true' followed by two 'false's. Yet, in a perfectly clear sense, every member of the infinite sequence makes the same claim and therefore they ought not to differ in truth-value, and this pulls us in the direction of holding (what Wittgenstein held in the case of tautologies and contradictions (1922, 5.43; see also $4.461,6.11)$ ) that they all say the same, namely nothing and hence are without truthvalue. This same tension arises for each of the family of paradoxes that we have been discussing. For example (to take the simplest) the Truth-Teller

\section{$\mathrm{T}$ : $\mathrm{T}$ yields a true statement.}

One could assign 'yields a truth' to T, but equally, one could assign 'yields a falsehood', so it may seem reasonable to suppose that $\mathrm{T}$ has a classical truth-value. But (especially in view of the fact that one cannot in a non-circular way say just what statement $\mathrm{T}$ yields), that there is no more reason to say that it is true than that it is false, pulls one in the direction of saying that it is without truth-value.

The resolution of this difficulty lies in reflecting that we typically state the truthconditions of a statement by means of a biconditional. Biconditionals are also frequently used for stating the rules of a game. Thus: 'A penalty point is awarded to a player iff his or her opponent intentionally impedes access to the ball'. Guided by such rules, a referee is able to adjudicate. Clearly the following biconditional would offer no guidance at all: 'A penalty is awarded to a player iff a penalty is awarded to a player'. I shall call such biconditionals 
vacuous, for the RHS fails to state any condition under which the LHS holds. For a given token sentence named ' $\mathrm{N}$ ', the truth-conditions of the statement yielded by $\mathrm{N}$ are supplied by the biconditional scheme:

$\mathrm{N}$ yields a true statement iff $\mathrm{w}$.

where ' $w$ ' abbreviates a token sentence typically of the same type as the one named ' $\mathrm{N}$ ' or of a translation thereof. ${ }^{11}$ Therefore, in attempting to give the truth-conditions for the truth-teller $\mathrm{T}$ we obtain, by substitution of $\mathrm{T}$ for $\mathrm{N}$ in the above scheme

$\mathrm{T}$ yields a true statement iff $\mathrm{T}$ yields a true statement.

a vacuous biconditional which fails to supply any truth-conditions for a use of T. But, as Mark Sainsbury points out, '[t]o contemplate an appropriate concatenation of words with understanding is to appreciate its truth-condition. There is no unanswered question about how the sentence manages to say something' (Sainsbury 1996, p.147). The point can be put like this: being a statement involves having a truth-condition. Now, as we have just seen, no nonempty truth-condition can be given for a use of $\mathrm{T}$; it suffers from what, in Section 3, we termed 'underspecification'. Hence T fails to yield a statement.

Vacuity of the sort that we have been discussing afflicts other members of the Liar family in which no direct self-reference occurs. Consider the pair
A: B yields a truth.
B: A yields a truth. 
The truth-condition for A (if it had one) would be

A yields a truth iff $B$ yields a truth.

And the truth-condition for B (if it had one) would be

$B$ yields a truth iff A yields a truth.

Solving these two biconditionals for A by transitivity gives the vacuous

A yields a truth iff A yields a truth.

so we conclude that A lacks content, delivers no truth-value, and so, likewise, does B.

G.E. Moore's 'Paradox of Analysis' can be read as a warning that giving a plausible general account of what makes an analytically true biconditional informative (non-vacuous) is going to be a non-trivial task. The above examples, however seem to be uncontroversial cases of vacuity: the meaning of a word is not explained merely by repeating that word; the truth-condition of an utterance cannot be given by announcing that it is true on condition that....it is true. Perhaps, so it may be objected, there are ways of specifying the content of an utterance other than by stating its truth-conditions. A child may come to understand the content of the assertion 'Here are five red apples', for example, by observing how competent speakers interact with the world, how they interweave a variety of actions when using those words to make that assertion (Wittgenstein 1953, $\S 1,7$ ). But there is nothing in or about the extra-linguistic world that discloses a content for T, A, or B. 
Less argument is needed in the case of paradoxes that are not truth-teller variants. Here, again, there is failure of specification, but of an even more pernicious kind. The following is surely a spectacularly flawed attempt to deliver a viable rule of squash: 'A let ball is awarded to a player iff a let ball is not awarded to a player'. Obviously, this 'rule' is empty and can offer no guidance to a referee. A 'rule' that cannot regulate is no rule. Likewise, there are 'definitions' that do not succeed in defining. The sentence 'Iens' can be a successful definition of 'Iendum' only if 'Iendum iff Iens' is true. But

$\mathrm{S}$ yields a true statement iff $\mathrm{S}$ does not yield a true statement.

is obviously not true. Therefore, by the same train of reasoning as we employed in the discussion of T, no truth-condition can be given for a use of the Strengthened Liar

S: S does not yield a true statement.

for the attempt to do so (above) is a biconditional that is not true. Hence the Strengthened Liar S fails to yield a statement and thus lacks a truth-value. I can say that, using a token (C) 'S does not yield a true statement', and the truth-condition of $\mathrm{C}$ is

C yields a true statement iff $\mathrm{S}$ does not yield a true statement

Since, as we have argued, $\mathrm{S}$ does not yield a true statement, $\mathrm{C}$ does.

J.L. Austin wrote that the Truth-teller is 'every bit as absurd' as the Liar (Austin 1950, p.122). But one might well be inclined to say that the Liar is even more absurd than the Truth-teller because its truth-conditions are not just vacuous but also visibly impossible to 
satisfy. If I take my telephone off the hook (the analogy works better with old-fashioned telephones) then one of the conditions necessary for my receiving an incoming call cannot be satisfied. That is why, if I dial my own number, there is no ringtone. But with my own 'phone on the hook, I can pick up another 'phone, dial exactly the same number, and. succeed in getting a ringtone. Cassationists will say that a certain token of 'This sentence yields an untrue statement' fails to yield a statement and hence be not true. And they can say so by using an equiform token, and be speaking the truth, if their 'This' points to that other token and the 'This' in that token points to itself.

The more complicated variants on the standard Liar paradox, such as the Epimenides, the Cretins and Yablo's Paradox are just more subtle ways of masking the fact that the sentences they contain are associated with vacuous truth-conditions and hence fail to yield statements. Our derivation, at the beginning of Section 3, of a contradiction from the Yablo sequence amounted just to a demonstration that the content of the first member, $\mathrm{Y}_{1}$, of that sequence is given by

$$
\mathrm{Y}_{1} \text { is true iff } \sim\left(\mathrm{Y}_{1} \text { is true }\right) \text {. }
$$

But a vacuous biconditional delivers no specification of content. It is this inadequate specification of content that is responsible for members of the generic Fibonacci having no numerical values and for the members of the Yablo sequence having no truth-values. Standing outside the sequence, we can use a token of the sentence 'For all integers $n>1, Y_{n}$ is not true.' to say something true, even though the equiform $\mathrm{Y}_{1}$ fails to state anything.

\section{Set-theoretic paradoxes}


Vacuity of biconditionals (aliter, failure to legitimately define) underlies other types of paradox. The biconditional

\section{$\mathrm{K} \varepsilon \mathrm{K}$ iff $\mathrm{K} \varepsilon \mathrm{K}$}

is vacuous, but is one of the conjuncts that appears when

\section{DefK: $\quad(\mathrm{x})(\mathrm{x} \varepsilon \mathrm{K}$ iff $\mathrm{x} \varepsilon \mathrm{x})$}

is expanded out as an infinite conjunction of its instantiations. Thus DefK fails to define a class $\mathrm{K}$; there is no class $\mathrm{K}$, but there is a 'near miss' class $\mathrm{K}$ ' defined by

\section{DefK $^{*}:($ For all $\mathrm{x}$, except $\mathrm{x}=\mathrm{K})(\mathrm{x} \varepsilon \mathrm{K}$ iff $\mathrm{x} \varepsilon \mathrm{x})$}

Likewise, no class R (the Russell Class) is given by

DefR: $(x)(x \in R$ iff $\sim(x \in x))$

although there is a 'near miss' class $\mathrm{R}^{*}$ in which, as in DefK", the range of the variable is restricted - in this case, so as not to include $\mathrm{R}^{*}$. (There are counterparts to these 'near miss' cases in the semantical paradoxes we have been considering. For example, no paradox would arise from Epimenides saying 'All Cretans except myself are total liars'.) In the set theoretical paradoxes, membership conditions cannot be formulated non-vacuously; in the semantical paradoxes, truth conditions cannot be formulated non-vacuously. Herein lies the 
deep similarity between these two families of paradox. The sets and the statements we thought we were dealing with are irredeemably ill-defined and so do not exist. ${ }^{12}$

Department of Philosophy, SECL

LAURENCE GOLDSTEIN

Cornwallis Building NW

University of Kent

Canterbury

Kent CT2 7NF

$U K$

L.Goldstein@kent.ac.uk

\section{References}

Almog, Joseph, J. Perry and H. Wettstein (eds) 1989: Themes from Kaplan. Oxford: Oxford University Press.

Austin, J.L. 1950: 'Truth'. Proceedings of the Aristotelian Society, supp. vol. 24, pp.111-28.

Chapuis, André and A. Gupta (eds) 2000: Circularity, Definition, and Truth. New Delhi: Indian Council of Philosophical Research.

De Rijk, L.M. 1966: 'Some Notes on the Mediaeval Tract De Insolubilibus, with the Edition of a Tract Dating from the End of the Twelfth Century'. Vivarium, 4, pp. 83-115.

Gaifman, Haim 1992: 'Pointers to Truth'. The Journal of Philosophy, 89, pp. 223-61.

2000: 'Pointers to Propositions', in Chapuis and Gupta, 2000, pp.79-121. (Also available at http://www.columbia.edu/ hg17/gaifman6.pdf).

Goldstein, Laurence 1999: 'Circular Queue Paradoxes — the Missing Link'. Analysis, 59, pp.284-90. 
Hughes, G.E. 1982: John Buridan on Self-Reference: Chapter Eight of Buridan's Sophismata. Cambridge: Cambridge University Press.

Kaplan, David 1977: 'Demonstratives: An Essay on the Semantics, Logic, Metaphysics, and Epistemology of Demonstratives and Other Indexicals', in Almog, Perry and Wettstein 1989, pp.565-614. Originally circulated in 1977.

Kripke, Saul 1975: 'Outline of a Theory of Truth'. The Journal of Philosophy, 72, pp. 690716.

Locke, John 1690: An Essay concerning Human Understanding, ed. P.H. Nidditch. Oxford: Clarendon Press 1979.

Maudlin, Tim 2004: Truth and Paradox: Solving the Riddles. Oxford: Oxford University Press.

Monk, Ray and A. Palmer (eds) 1996: Bertrand Russell and the Origins of Analytical Philosophy. Bristol: Thoemmes Press.

Priest, Graham, JC Beall and B. Armour-Garb 2004: The Law of Non-Contradiction: New Philosophical Essays. Oxford: Oxford University Press.

Putnam, Hilary 1982: Reason, Truth and History. Cambridge: Cambridge University Press.

Sainsbury, Mark 1996: 'How Can We Say Something?', in Monk and Palmer 1996, pp.13751.

Spade, Paul 1987: 'Five Early Theories in the Mediaeval Insolubilia-Literature'. Vivarium, 25, pp.24-46.

Tennant, Neil 2004: ‘An Anti-realist Critique of Dialetheism', in Priest, Beall and ArmourGarb, pp.355-84.

Wittgenstein, Ludwig 1922: Tractatus Logico-Philosophicus. London: Routledge and Kegan Paul.

Wittgenstein, Ludwig 1953: Philosophical Investigations. Oxford: Basil Blackwell. 
Yablo, Stephen 1985: 'Truth and Reflection'. Journal of Philosophical Logic, 14, pp.297349.

Yablo, Stephen 1993: 'Paradox without Self-reference'. Analysis, 53, pp. 252-3. 
${ }^{1}$ For information on the Fibonacci numbers, the Golden Section and the Golden String, consult Ron Knott's impressive web site http://www.mcs.surrey.ac.uk/Personal/R.Knott/Fibonacci/fib.html.

2 For example, Wittgenstein $(1922,4.461,4.4611,6.1,6.11,6.111,6.1264)$ argued that, though tautologies and contradictions are perfectly grammatical sentences (Satzzeichen) and are not nonsensical (unsinnig), they are without sense (sinnlos), they 'say nothing'; in other words, they do not yield statements (Sätze).

${ }^{3}$ This would be the outcome of applying the evaluation scheme of Kripke (1975).

${ }^{4}$ Following, respectively, the semantic schemes of Maudlin (2004) or that of Gaifman (1992, 2000). The approach taken here is closer to the 'pointer semantics' of Gaifman, in which the bearers of truth are inscriptions (or other such physical objects) as they are used, and I shall henceforth follow Gaifman in referring to lack of truth-value as 'GAP'.

${ }^{5}$ J.L. Austin (1950, p.127) raises this issue in a characteristically chirpy way: 'When is a statement not a statement?'. Of the many suggested answers, he includes '[w]hen it is a formula in a calculus: when it is a performatory utterance; when it is a value judgement: when it is a definition: when it is part of a work of fiction'. Other cases for which it has been claimed that a meaningful sentence fails to deliver a statement include instances of presupposition-failure, and of sentences containing demonstrative expressions unaccompanied by a demonstration. We shall later be suggesting the answer: "When the candidate statement fails to have a truth-condition'. Austin's view was that, when something masquerading as a statement has been unmasked, it is better ' $n o t$ to call it a statement and not to say that it is true or false'.

${ }^{6}$ Dialetheists, of course, disagree but their position is put under irresistible pressure by Tennant (2004), and I shall not discuss it here. 
${ }^{7}$ As Kripke (1975, p.693) points out, if 'Jack' is not already a name in the language we are using, then there is nothing to prevent us giving the name 'Jack' to the uninterpreted finite sequence of marks 'Jack is short', but, as he notes, this does not show that a similar technique can be employed to obtain directly self-referential propositions.

8 An identical point is made by Buridan in his Eighth Sophism, where he is discussing the situation in which Socrates says 'What Plato is saying is false' while Plato says 'What Socrates is saying is false'. Buridan comments: 'There is no more reason why Socrates' proposition should be true, or false, than Plato's, or vice versa, since they stand in an exactly similar relation to each other. So I shall assume that if either is true, so is the other, and if either is false, so is the other.' Buridan goes on to conclude that the propositions of both Socrates and Plato are false (Hughes 1982, pp.51-2).

${ }^{9}$ This important distinction between the sentence proferred (prolatum) in an utterance and the thought expressed (assertio cum prolatione) was made as early as the early thirteenth century by the anonymous author of a treatise called Insolubilia Monacensia. See de Rijk (1966, p.106). A similar view is expressed by John Locke: 'Man therefore had by Nature his Organs so fashioned, as to be fit to frame articulate Sounds, which we call Words. But this was not enough to produce Language; for Parrots, and several other Birds, will be taught to make articulate Sounds distinct enough, which yet, by no means, are capable of Language. Besides articulate Sounds, therefore, it was farther necessary, that he should be able to use these Sounds, as Signs of internal Conceptions; and to make them stand as marks for the Ideas within his own mind' (Locke 1690, Bk 3, Ch..1, Sect. 1-2). (I wish to suggest that these authors identified an important distinction, not to suggest that they offered a sustainable account of it.)

${ }^{10}$ For a defence of the claim that the words in a sentence do not intrinsically possess semantical properties, see Putnam (1982, p.5). The relevant section also available at 
http://www.uwichill.edu.bb/bnccde/ph29a/putnam.html. Elucidating this point was a preoccupation of Wittgenstein's, early and late. See, for example, (1922, 3.1-3.12) and $(1953, \S \S 260-1)$.

${ }^{11}$ Various caveats need to be entered. Any referring expression in $\mathrm{N}$ must co-refer with its counterpart in ' $w$ '. When $\mathrm{N}$ contains an indexical expression, then ' $\mathrm{w}$ ' will typically not be of the same type as $\mathrm{N}$, since systematic adjustments need to be made. For example, if $\mathrm{N}$ contains the first-person pronoun 'I', then ' $w$ ' will contain instead 'The speaker of N'.

${ }^{12}$ Comments from Max Deutsch, Chad Hansen and Joe Lau led me to repair a weakness in an earlier version of this paper and I am grateful also to an anonymous referee who identified two points that needed sharpening. 